
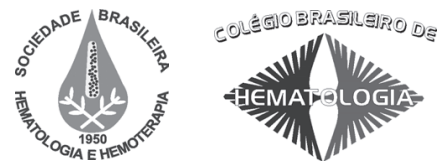

\title{
Anemia hemolítica microangiopática induzida por tacrolimus e ciclosporina $\mathbf{A}$
}

\section{Microangiopathic hemolytic anemia induced by tacrolimus and ciclosporine $A$}

Rafael S. Vasconcelos ${ }^{1}$

José Zanis Neto ${ }^{2}$

Mônica Parolin ${ }^{3}$

Júlio Coelho ${ }^{4}$

Paulo G. O. Müller
Relatamos caso raro de anemia hemolítica microangiopática em paciente após transplante hepático por cirrose Child B causada por deficiência de alfal-antitripsina. Após análise clínica e laboratorial extensa, concluiu-se que o quadro de hemólise foi causado pela medicação imunossupressora utilizada após transplante. Inicialmente foi utilizado tacrolimus que, posteriormente, foi substituido por ciclosporina A, entretanto não houve melhora da hemólise. Após suspensão das duas drogas, houve melhora importante clínica do paciente, com normalização dos parâmetros hematológicos. Rev. Bras. Hematol. Hemoter. 2008;30(6):505-506.

Palavras-chave: Anemia hemolitica microangiopática; tacrolimus; transplante.

\section{Introdução}

Tacrolimus (FK506) é um macrolídeo com potente atividade imunossupressora e representou um importante avanço no controle de receptores de órgãos. Apesar de FK506 e ciclosporina $\mathrm{A}(\mathrm{CyA})$ serem totalmente diferentes quanto à estrutura e se ligarem a proteínas citosólicas diferentes, ambos exercem uma atividade antilinfócito seletiva in vitro. Ambos inibem a síntese de interleucina-2 (IL-2) e outras citocinas (IL-3, IL-4, fator de necrose tumoral e interferon gama) no nível de transcrição do RNAm, levando a uma ação seletiva de supressão da função da célula $T$.

FK506 e CyA compartilham algumas propriedades farmacológicas e efeitos colaterais: nefrotoxicidade, neurotoxicidade, diabetogênese. Anemia hemolítica microangiopática é uma complicação bem documentada de CyA após transplante de células-tronco hematopoéticas ou órgãos sólidos, mas esse evento raramente foi associado com FK506, havendo poucos casos documentados em literatura. Relatamos episódio de anemia hemolítica microangiopática em receptor de órgão sólido, com análises de parâmetros hematológicos e biológicos sugerindo FK506 e CyA como causadores prováveis de anemia hemolítica.

\section{Relato do Caso}

Paciente masculino, 52 anos, recebeu transplante hepático em 20 de setembro de 2002 por cirrose hepática Child $\mathrm{B}$ por deficiência de alfa1-antitripsina. Terapia imunossupressora inicial consistia de FK506 $18 \mathrm{mg} /$ dia e prednisona $15 \mathrm{mg} /$ dia. Em 15 de outubro de 2002 foi internado no Hospital de Clínicas - UFPR por quadro de anemia grave sintomática. Hemograma na ocasião mostrava: Hb: 7,5 g/dL, Vg: 22\%, VCM: 82,7 , leucócitos: $5,85 \times 10^{9} / \mathrm{L}$, neutrófilos: $3,97 \times 10^{9} / \mathrm{L}$, plaquetas: $63 \times 10^{9} / \mathrm{L}$, reticulócitos: $10,7 \%$. Na análise do esfregaço sanguíneo, notava-se anisocitose, poiquilocitose, policromatofilia importante, presença de micrócitos, ovalócitose e alguns esquistócitos por campo de maior aumento. Exames laboratoriais mostravam: LDH 415 U/1, bilirrubina total: $5,86 \mathrm{mg} / \mathrm{dL}$, bilirrubina indireta: $4,53 \mathrm{mg} / \mathrm{dL}$, TGO (AST): $11 \mathrm{u} / 1$, TGP (ALT): $28 \mathrm{u} / 1$, dosagem tacrolimus: $8,0 \mathrm{ng} / \mathrm{mL}$, Coombs direto: negativo, Coombs indireto: negativo, Ham: negativo, sacarose: negativo, teste de fragilidade osmótica negativo, eletroforese de hemoglobina normal, triagem para deficiência de G6PD negativa, antigenemia para citomegalovírus negativa. Análise de medula óssea evidenciou hiperplasia de linhagem eritróide.

${ }^{1}$ Residente em Hematologia do HC-UFPR-PR.

${ }^{2}$ Chefe do Serviço de Transplante de Medula Óssea do HC-UFPR-PR.

${ }^{3}$ Chefe do Serviço de Transplante Hepático do HC-UFPR-PR.

${ }^{4}$ Chefe do Serviço de Cirurgia do Aparelho Digestivo do HC-UFPR-PR.

${ }^{5}$ Médico do Hospital de Clínicas - UFPR-PR.

Hospital de Clínicas - Universidade Federal do Paraná - UFPR - Curitiba-PR.

Correspondência: Rafael de Sá Vasconcelos

Rua Nilo Cairo 524, apto 204

80060-050 - Curitiba-PR - Brasil_Email:rafasabr@yahoo.com.br 
Realizou-se teste terapêutico com elevação da dosagem de prednisona para $60 \mathrm{mg} /$ dia, sem melhora. Substituiu-se então imunossupressor FK506 por CyA na dose de $400 \mathrm{mg} /$ dia em 06 de novembro de 2002, com paciente apresentando melhora clínica importante. Hemograma do dia 13 de novembro mostrava: Hb: 9,0g/dL, Vg: 25,9\%. Em 22 de novembro foi realizada dosagem de haptoglobina, cujo resultado foi de $8,0 \mathrm{mg} / \mathrm{dL}$, comprovando existência de hemólise.

Houve agravamento do quadro de anemia hemolítica, com hemograma do dia 03 de dezembro de 2002 mostrando $\mathrm{Hb}: 6,5 \mathrm{~g} / \mathrm{dL}, \mathrm{Vg}$ : 19,8\% e reticulócitos: $13,6 \%$. O paciente foi submetido a transfusão de concentrado de hemácias e optou-se pela troca da CyA $400 \mathrm{mg} / \mathrm{dia}$ por micofenolato mofetil $2 \mathrm{~g} / \mathrm{dia}$. Análises efetuadas a seguir demonstraram melhora do quadro da anemia hemolítica microangiopática, sugerindo a confirmação da hipótese de hemólise por uso de drogas. Exames do dia 6 de janeiro de 2003 mostravam: $\mathrm{Hb}: 11,6 \mathrm{~g} / \mathrm{dL}, \mathrm{Vg}: 33,2 \%$, reticulócitos: 4,1\%, com ausência de esquistócitos em esfregaço sanguíneo.

Atualmente o paciente apresenta quadro de anemia hemolítica compensada, com terapia imunossupressora constituída por sirulimus $2 \mathrm{mg} / \mathrm{dia}$, micofenolato mofetil $2 \mathrm{~g} / \mathrm{dia}$, deflazacort $6 \mathrm{mg} / \mathrm{dia}$. Exames de dezembro de 2007 mostram: $\mathrm{Hb} 15,8 \mathrm{~g} / \mathrm{dL}, \mathrm{Vg}: 49,3 \%$, leucócitos de 4,2 x 10\%/L, neutrófilos: $3,2 \times 10^{9} / \mathrm{L}$, plaquetas: $87 \times 10^{9} / \mathrm{L}$, bilirrubina total: $2,0 \mathrm{mg} / \mathrm{dL}$, bilirrubina indireta: $1,7 \mathrm{mg} / \mathrm{dL}, \mathrm{LDH}: 445 \mathrm{U} / \mathrm{ml}$, haptoglobina: $56,4 \mathrm{mg} / \mathrm{dL}$.

\section{Discussão}

As anemias hemolíticas são um grupo de doenças no qual a sobrevida das células vermelhas é reduzida periodicamente ou continuamente. São geralmente classificadas de acordo com a presença de algum defeito intrínseco das hemácias ou de fator externo. Anemias hemolíticas por fator externo são imunes ou microangiopáticas.

As anemias hemolíticas microangiopáticas são um grupo de doenças nas quais há fragmentação das células vermelhas. A fragmentação hemolítica ocorre quando forças mecânicas rompem a integridade física da membrana eritrocitária. Diversas drogas são associadas com hemólise microangiopática, como ciclosporina, tacrolimus, quinino, clopidogrel, ticlopidina, mitomicina $\mathrm{C}$, penicilina, diclofenaco, interferon. O tratamento nessa situação é a suspensão da droga suspeita. O papel da plasmaférese é incerto, mas esse tratamento pode ser apropriado, considerando a alta morbidade e mortalidade do quadro.

Em conexão com transplante de órgãos, a anemia hemolítica pode ter diferentes etiologias: rejeição do órgão, ciclosporina e infecções. No caso do paciente em questão, a causa mais provável do quadro hemolítico, que ocorreu precocemente ao transplante, parece estar associada ao uso de tacrolimus, que se encontrava em nível terapêutico. Posteriormente o paciente desenvolveu um quadro de anemia hemolítica grave após uso da ciclosporina.

$\mathrm{O}$ reconhecimento do quadro de anemia hemolítica microangiopática associada a drogas é importante para evitar a reexposição à droga suspeita e doença recorrente.

\begin{abstract}
We report a rare case of acute hemolysis in a patient after liver transplant for Child B cirrhosis caused by alfal-antitripsine deficiency. History and laboratorial examinations indicated that the most probable cause of the hemolysis was the use of the immunosupressor tacrolimus and later Cyclosporine A. After the discontinuation of these drugs, there was improvement in the clinical condition of the patient, with a compensated hemolytic anemia. Rev. Bras. Hematol. Hemoter. 2008;30(6):505-506.
\end{abstract}

Key words: Microangiopathic hemolytic anemia; tacrolimus; transplant.

\section{Referências Bibliográficas}

1. Hirose R, Vincenti F. Immunosuppression: today, tomorrow, and withdrawal. Semin Liver Dis. 2006;26(3):201-10.

2. Mach-Pascual S, Samii K, Beris P. Microangiopathic hemolytic anemia complicating FK506 (tacrolimus) therapy. Am J Hematol. 1996;52(4):310-2.

3. DiGiuseppe JA, Bastacky SI, Shirey RS, Silberman MA, Hutchins GM, Ness PM. Tacrolimus-related posttransplant lymphoproliferative disorder presenting as autoimmune hemolytic anemia. Arch Pathol Lab Med. 1996;120(3):282-5.

4. Abu-Elmagd KM, Bronsther O, Kobayashi M, Yagihashi A, Iwaki $\mathrm{Y}$, Fung $\mathrm{J}$ et al. Acute hemolytic anemia in liver and bone marrow transplant patients under FK 506 therapy. Transplant Proc. 1991; 23(6):3190-2.

5. Tabbara IA. Hemolytic anemias. Diagnosis and management. Med Clin North Am. 1992;76(3):649-68.

6. Medina PJ, Sipols JM, George JN. Drug-associated thrombotic thrombocytopenic purpura-hemolytic uremic syndrome. Curr Opin Hematol. 2001;8(5):286-93.

7. Danesi R, Del Tacca M. Hematologic toxicity of immunosuppressive treatment. Transplant Proc. 2004;36(3):703-4.

8. Oteo JF, Alonso-Pulpón L, Diez JL, Camacho C, Expósito C, González A et al. Microangiopathic hemolytic anemia secondary to cyclosporine therapy in a heart and liver transplant recipient. J Heart Lung Transplant. 1996;15(3):322-4.

9. Maheshwari A, Mishra R, Thuluvath PJ. Post-liver-transplant anemia: etiology and management. Liver Transpl. 2004;10(2):165-73

10. Alonso-Santor JE, Gutierrez-Zufiaurre JL, Pérez-Castrillón JL. Microangiopathic hemolytic anemia secondary to cyclosporine. Ann Pharmacother. 2004;38(5):903.

Avaliação: Editor e dois revisores externos

Conflito de interesse: não declarado

Recebido: 21/07/2008

Aceito após modificações: 12/10/2008 